\title{
Effect of tellurium promoter on vanadium phosphate catalyst for partial oxidation of n- Butane.
}

\begin{abstract}
Te-promoted (1\%) vanadium phosphate catalyst (VPDTe) was prepared via VOPO4.2H2O by calcining its precursor VOHPO4. $0.5 \mathrm{H} 2 \mathrm{O}$ in a flow of n-butane/air. VPDTe catalyst has resulted a higher existence of V5+ phase with V5+/V4+ ratio of 0.23. SEM micrographs show that Te addition altered the arrangement of the platelets from "rose-like" clusters to layer with irregular shape. Te addition has also markedly lowered the reduction activation energies of the vanadium phosphate catalyst as revealed by TPR profile. The amount of active oxygen species associated with $\mathrm{V} 4+$ phase of the Te promoted catalyst was significantly higher than those of the unpromoted catalyst. These observations suggest that high mobility and availability of reactive oxygen species contributed to the enhancement of n-butane conversion up to $80 \%$ at $673 \mathrm{~K}$, while only $47 \%$ over unpromoted catalyst $(2400$ $\mathrm{h}-1,1.7 \%$ n-butane in air).
\end{abstract}

Keyword: Vanadium phosphate; Tellurium; Pomoter; Butane oxidation. 